Journal of Low Temperature Physics, Vol. 138, Nos. 3/4, February 2005 (@ 2005)

DOI: $10.1007 / \mathrm{s} 10909-005-2269-0$

\title{
Vortices in trapped boson-fermion mixtures
}

\author{
M. E. Taşgın, A. L. Subaşı, M. Ö. Oktel, and B. Tanatar \\ Department of Physics, Bilkent University, Bilkent, 06800 Ankara, Turkey
}

We consider a trapped system of atomic boson-fermion mixture with a quantized vortex. We investigate the density profiles of bosonic and fermionic components as functions of the boson-boson and boson-fermion short-range interaction strengths within the mean-field approach. Stability of a vortex and conditions for the phase segregation are studied. We compare and contrast our results with the related system of droplets of ${ }^{3} \mathrm{He}-{ }^{4} \mathrm{He}$ mixtures.

PACS numbers: 05.30.-d, 03.75.Ss, 67.60.-g, 67.40.Vs.

\section{INTRODUCTION}

After the successful achievement of Bose-Einstein condensation in dilute alkali gases ${ }^{1}$ under magneto-optical trap potentials, a vast theoretical and experimental activity on cold degenerate quantum gases has followed. ${ }^{2}$ More recently, fermionic gases are cooled to quantum degeneracy temperatures facilitated by mixing with cold bosonic gases by a process known as sympathetic cooling. Experimental progress in this direction has culminated in achieving the realization of quantum degenerate Bose-Fermi mixtures by several groups. ${ }^{3-8}$ Currently there are a number of experiments on bosonfermion mixtures in harmonic traps. In the Paris experiment ${ }^{4}{ }^{6} \mathrm{Li}-{ }^{7} \mathrm{Li}$ mixture with a repulsive boson-fermion scattering length, and in the Florence experiment ${ }^{8}{ }^{40} \mathrm{~K}-{ }^{87} \mathrm{Rb}$ mixture with an attractive boson-fermion scattering length are realized. Furthermore, using Feshbach resonances many groups have tuned the scattering length both for bosons and fermions. Theoretical studies on trapped boson-fermion mixtures employed the mean-field theory at zero temperature to determine the density profiles of respective components. ${ }^{9-12}$ Related properties such as the stability against phase separation and collapse were also investigated. ${ }^{13,14}$ The temperature effects and their role in phase separation were addressed by Akdeniz et al. ${ }^{15}$ The critical temperature of the Bose-Einstein condensation in a trapped mixture were 
considered by several groups. ${ }^{16,17}$

Motivated by these recent experiments on boson-fermion mixtures of dilute alkali gases, in this paper, we study the ground state properties of such system in the presence of a single vortex. The quantized vortices are important in establishing the superfluid nature of Bose condensates. ${ }^{2}$ Recently there has been numerous experimental works devoted to the creation and investigation of properties of quantized vortices in trapped condensates. ${ }^{18}$ We are also motivated by the analogies and differences of trapped quantum gases and Helium droplets as prototypes of finite quantum fluids as recently surveyed by Dalfovo and Stringari. ${ }^{19}$ To this end, we make contact with recent theoretical calculations of a vortex state in ${ }^{3} \mathrm{He}-{ }^{4} \mathrm{He}$ droplets. ${ }^{20-22}$

We employ the mean-field theory at zero temperature to consider a mixture of Bose condensed atoms and spin-polarized gas of fermions in a harmonic trap. Introducing a single quantized vortex through the FeynmanOnsager ansatz we study the ensuing density profiles of respective species. The density profiles are obtained by solving the mean-field equations for the trapped boson-fermion mixture using a variational ansatz.

\section{MODEL AND THEORY}

We consider $N_{B}$ bosons of mass $m_{B}$ in the condensed state and $N_{F}$ fermions of mass $m_{F}$ in respective trap potentials $V_{B}=\frac{1}{2} m_{B} \omega_{B}^{2} r^{2}$ and $V_{F}=\frac{1}{2} m_{F} \omega_{F}^{2} r^{2}$ in the form of isotropic harmonic oscillators. $\omega_{B}$ and $\omega_{F}$ are the trap frequencies for bosonic and fermionic species, respectively. The ground-state energy functional of a mixture of bosons and fermions in the mean-field approximation is given by

$$
E\left[n_{B}(r), n_{F}(r)\right]=\int d \mathbf{r}\left(E_{B}+E_{F}+E_{B F}\right) .
$$

The energy density of bosons is

$$
E_{B}=\frac{\hbar^{2}}{2 m_{B}}|\nabla \Psi(r)|^{2}+V_{B}(r) n_{B}(r)+\frac{g}{2} n_{B}(r)^{2},
$$

where $\Psi(r)$ is the condensate wavefunction, $n_{B}(r)=|\Psi(r)|^{2}$ is the condensate density distribution, and $g$ is the boson-boson interaction strength. Since the fermions are assumed to be noninteracting, we have

$$
E_{F}=T_{F}\left[n_{F}(r)\right]+V_{F}(r) n_{F}(r),
$$

where the kinetic energy functional for fermions with single spin species in the Thomas-Fermi approximation ${ }^{9,10,14}$ is $T_{F}=\left(6 \pi^{2} n_{F}\right)^{5 / 3} / 20 \pi^{2} m_{F}$ and 
the fermion density distribution is $n_{F}(r)=\frac{(2 m)^{3 / 2}}{6 \pi^{2}}\left[\varepsilon_{F}-V_{F}(r)-h n_{B}(r)\right]^{3 / 2}$ with $\varepsilon_{F}$ the Fermi energy. The boson-fermion interaction energy density is $E_{B F}=h n_{F}(r) n_{B}(r)$, where $h$ is the boson-fermion interaction strength. The total energy-density for the mixture now becomes

$$
\begin{gathered}
E\left[n_{B}, n_{F}\right]=\int d^{3} r\left[\frac{\hbar^{2}}{2 m_{B}}|\nabla \Psi(r)|^{2}+V_{B}(r)|\Psi(r)|^{2}+\frac{g}{2}|\Psi(r)|^{4}\right. \\
\left.T_{F}\left(n_{F}\right)+V_{F}(r) n_{F}(r)+h n_{F}(r)|\Psi(r)|^{2}\right] .
\end{gathered}
$$

We have assumed that the fermionic component of the mixture is spinpolarized whereby the $s$-wave scattering between the fermions is inhibited by the Pauli principle. $g$ and $h$ are the boson-boson and boson-fermion interaction strengths, respectively, related to the $s$-wave scattering lengths $a_{B B}$ and $a_{B F}$ as measured in experiments, ${ }^{4,5}$ viz. $g=4 \pi \hbar^{2} a_{B B} / m_{B}$ and $h=4 \pi \hbar^{2} a_{B F} / \mu_{B F}$, where $\mu_{B F}$ is the reduced mass. We introduce a quantized vortex through the Feynman-Onsager ansatz, $\Psi(r)=\psi(r) e^{i \phi}$, which amounts to adding a centrifugal energy term $\frac{\hbar^{2}}{2 m_{B} r^{2}}|\psi|^{2}$ to the total energy functional. Our goal is to minimize the total energy functional subject to the normalization conditions $\int d \mathbf{r} n_{B}(r)=N_{B}$ and $\int d \mathbf{r} n_{F}(r)=N_{F}$.

To study the density profiles of boson and fermion components of the mixture, we now introduce the variational wavefunction for the condensate with a vortex, $\Psi(r, \phi)=A r e^{i \phi} e^{-\alpha r^{2}}$ where $A$ is the normalization constant and $\alpha$ is the variational parameter. The normalization integral for $N_{B}$ bosons yields $A=N_{B}^{1 / 2}\left(128 \alpha^{5} / 9 \pi^{3}\right)$.

\section{RESULTS AND DISCUSSION}

We have minimized the total energy of the mixture with respect to the variational parameter $\alpha$ and the fermion density $n_{F}(r)$ using the number of particles $N_{B}$ and $N_{F}$ as constraints. We have assumed the same mass $m_{B}=m_{F}$ for both species and the same trap frequency $\omega_{B}=\omega_{F}$ for simplicity. The boson-boson and boson-fermion interaction strengths are treated as tunable parameters.

In Fig. 1 we show the density profile of fermion species $n_{F}(r)$ as a function of the radial coordinate for various values of the repulsive bosonfermion interaction strength. For fixed boson-boson repulsive interaction ( $g=0.005 \hbar \omega a_{H O}^{3}$ in the examples shown) we observe a depletion in the central region of the fermion density as boson-fermion interaction strength increases. The dip in the central region of $n_{F}(r)$ coincides with the maximum of the density profile of the condensate with a vortex. Further increase in $h$ 


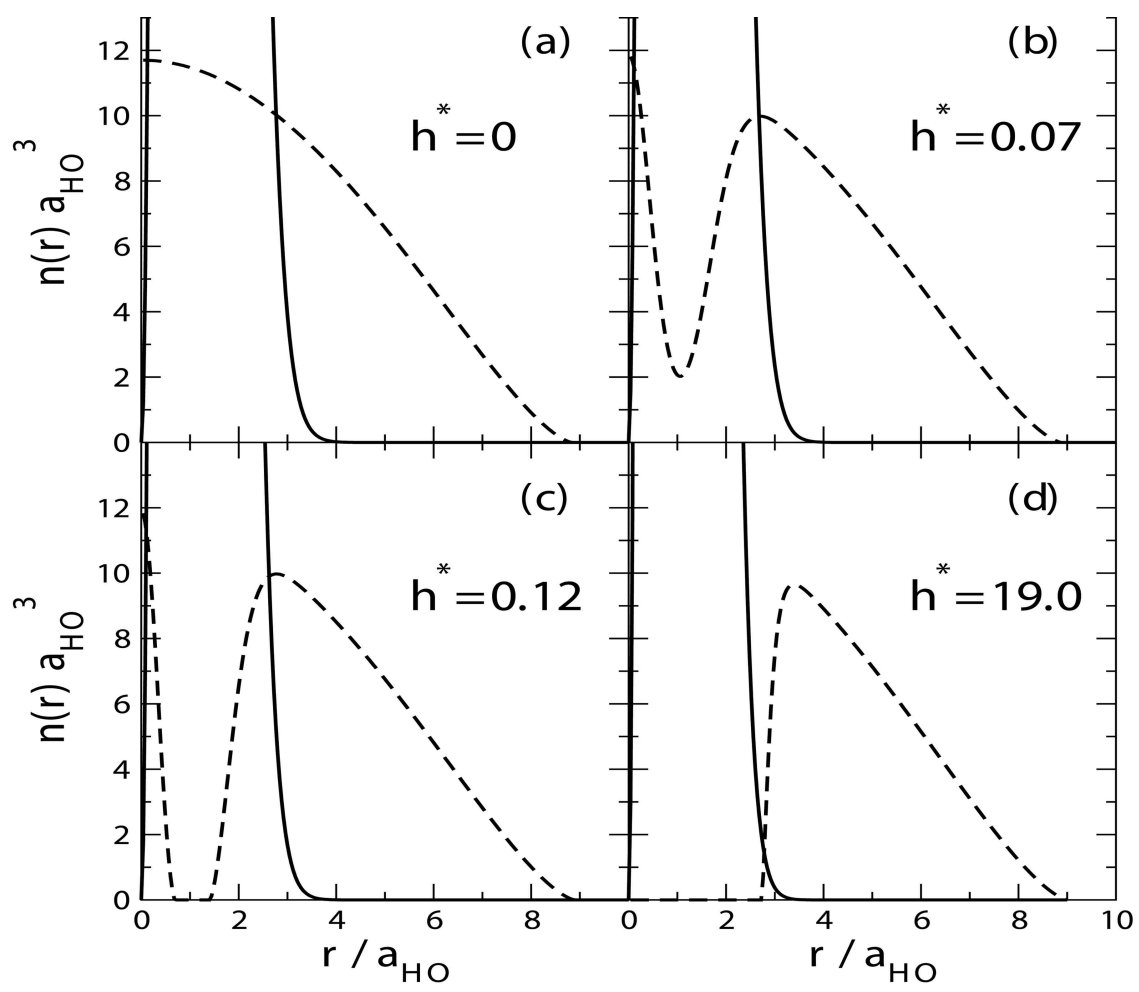

Fig. 1. Density profile of fermions $n_{F}(r)$ (dashed lines) for $g=0.005 \hbar \omega a_{H O}^{3}$ and $N_{B}=N_{F}=10^{4}$ particles. $h^{*}=h /\left(\hbar \omega a_{H O}^{3}\right)$. Solid lines indicate the boson density $n_{B}(r)$.

causes the break up of fermion density into two parts, one filling the vortex core, the other part pushed to the outer region [Fig.1(c)]. Eventually, when $h$ becomes very large, the fermions disappear from the vortex core region and occupy only the outer region surrounding the condensate [Fig.1(d)]. This last situation is the phase separated case of two species, similar to the theoretically calculated case of trapped boson-fermion mixtures without a vortex. ${ }^{6,7}$ The small overlap of boson $n_{B}(r)$ and fermion $n_{F}(r)$ densities is an artifact of Gaussian variational wavefunction which would give in to a complete phase segregation in more elaborate calculations.

We point out the similarity between our results shown in Fig. 1 and those of Mayol et al. ${ }^{20}$ who considered quantized vortices in ${ }^{3} \mathrm{He}-{ }^{4} \mathrm{He}$ droplets. They have found that even a small number of ${ }^{3} \mathrm{He}$ atoms fills the vortex 


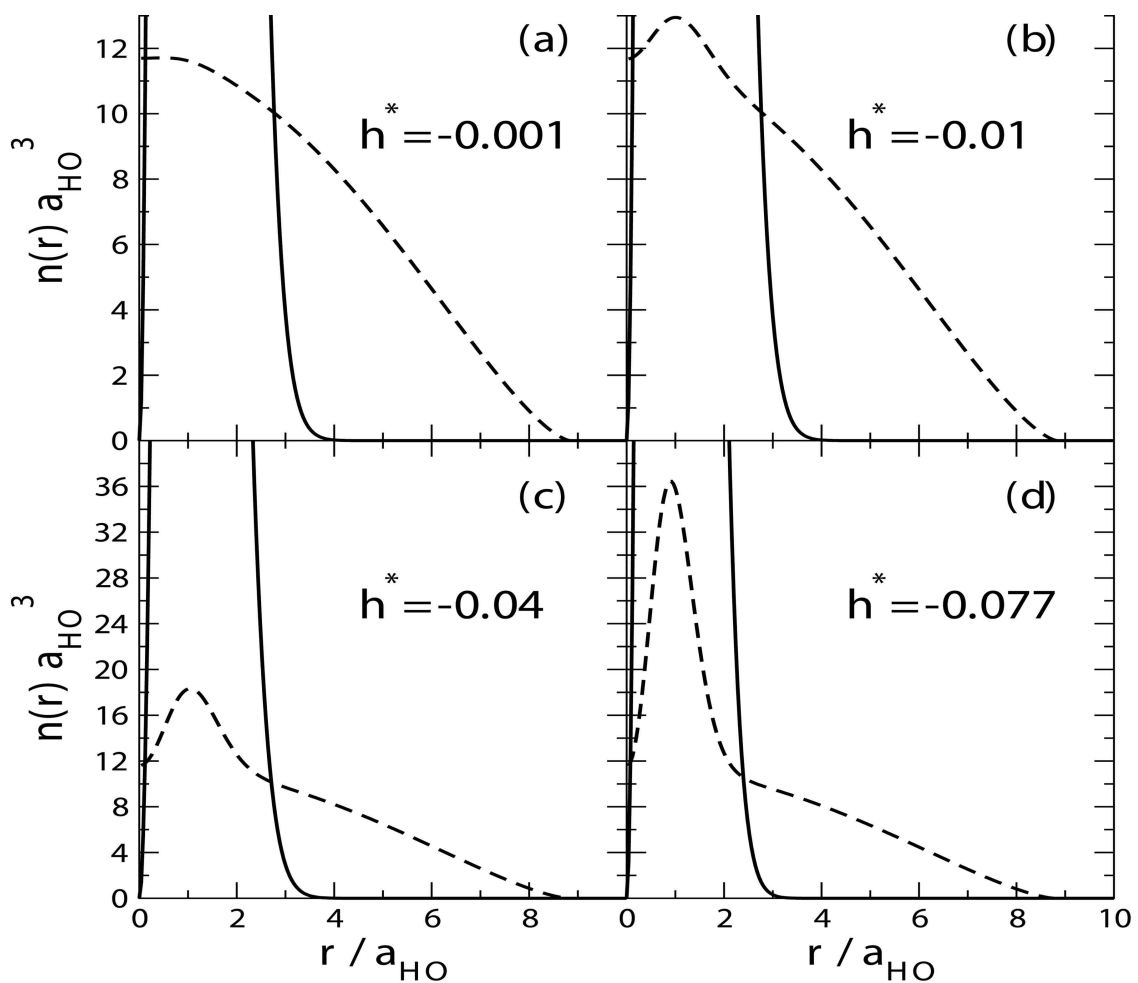

Fig. 2. Density profile of fermions $n_{F}(r)$ (dashed lines) for $g=0.005 \hbar \omega a_{H O}^{3}$ and $N_{B}=N_{F}=10^{4}$ particles. $h^{*}=h /\left(\hbar \omega a_{H O}^{3}\right)$. Solid lines indicate the boson density $n_{B}(r)$.

core provided by the quantized vortex in a ${ }^{4} \mathrm{He}$ condensate. Whereas in the case of ${ }^{3} \mathrm{He}-{ }^{4} \mathrm{He}$ mixtures the strong interaction potential between $\mathrm{He}$ atoms is fixed, the interactions between the alkali atoms can be tuned by Feshbach resonances to study a wider range of density profiles and possible phase separations.

We next consider attractive interactions between bosons and fermions. As shown in Fig. 2 an attractive boson-fermion interaction strength causes the central region of the fermion density $n_{F}(r)$ to increase. At a critical value of $h$ the system becomes unstable and the fermionic component collapses much like the situation in vortex-free boson-fermion mixtures studied previously. ${ }^{7,8}$

Our variational calculations employing a Gaussian ansatz may be im- 
proved by choosing better variational wavefunctions or numerically solving the coupled Euler-Lagrange equations for the mixture. We surmise, however, the results reported here should be qualitatively correct.

\section{ACKNOWLEDGMENTS}

This work was partially supported by TUBITAK, by NATO under Grant No. SfP971970, by the Turkish Academy of Sciences (TUBA), and by the Turkish Department of Defense under Grant No. KOBRA-002.

\section{REFERENCES}

1. M. H. Anderson, J. R. Ensher, M. R. Matthews, C. E. Wieman, and E. A. Cornell, Science 269, 198 (1995); K. B. Davis, M.-O. Mewes, M. R. Andrews, N. J. van Druten, D. S. Durfee, D. M. Kurn, and W. Ketterle, Phys. Rev. Lett. 75, 3969 (1995).

2. F. Dalfovo, S. Giorgini, L. P. Pitaevskii, and S. Stringari, Rev. Mod. Phys. 71, 463 (1999); A. J. Leggett, ibid. 73, 307 (2001).

3. S. G. Crane, X. Zhao, W. Taylor, and D. J. Vieira, Phys. Rev. A 62, 011402 (2000).

4. F. Schreck et al. Phys. Rev. Lett. 87, 080403 (2001).

5. J. Goldwin, S. B. Papp, B. DeMarco, and D. S. Jin, Phys. Rev. A 65, 021402 (2002).

6. Z. Hadzibabic et al. Phys. Rev. Lett. 88, 160401 (2002).

7. G. Ferrari et al. Phys. Rev. Lett. 89, 053202 (2002).

8. G. Roati et al. Phys. Rev. Lett. 89, 150403 (2002).

9. K. Mølmer, Phys. Rev. Lett. 80, 1804 (1998).

10. N. Nygaard and K. Mølmer, Phys. Rev. A 59, 2974 (1999).

11. T. Miyakawa, T. Suzuki, and H. Yabu, Phys. Rev. A 64, 033611 (2001); T. Miyakawa, K. Oda, T. Suzuki, and H. Yabu, J. Phys. Soc. Jpn. 69, 2779 (2000).

12. M. Amoruso, A. Minguzzi, S. Stringari, M. P. Tosi, and L. Vichi, Eur. Phys. J. D 4, 261 (1998).

13. R. Roth and H. Feldmeier, Phys. Rev. A 65, 021603 (2002).

14. R. Roth, Phys. Rev. A 66, 013614 (2002).

15. Z. Akdeniz et al. J. Phys. B 35, L105 (2002); Phys. Rev. A 66, 013620 (2002).

16. Y.-L. Ma and S.-T. Chui, Phys. Rev. A 66, 053611 (2002).

17. A. P. Albus et al. J. Phys. B 35, L551 (2002).

18. K. W. Madison et al. Phys. Rev. Lett. 84, 806 (2000); B. P. Anderson et al. ibid. 85, 2857 (2000); J. R. Abo-Shaeer et al. Science 292, 476 (2001).

19. F. Dalfovo and S. Stringari, J. Chem. Phys. 115, 10078 (2001).

20. R. Mayol, M. Pi, and M. Barranco, Phys. Rev. Lett. 87, 145301 (2001).

21. F. Dalfovo, R. Mayol, M. Pi, and M. Barranco, Phys. Rev. Lett. 85, 1028 (2000).

22. H. Shi and S. T. Chui, Physica B 284-288, 166 (2003). 